\title{
An Improved Differential Evolution Algorithm for a Multicommodity Location-Inventory Problem with False Failure Returns
}

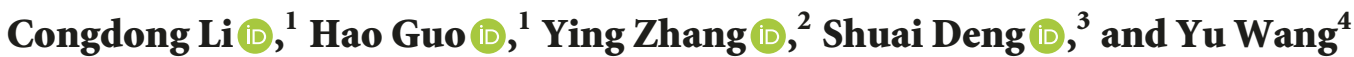 \\ ${ }^{1}$ School of Management, Jinan University, Guangzhou, Guangdong 510632, China \\ ${ }^{2}$ Department of Industrial and Systems Engineering, State University of New York at Buffalo, Buffalo, NY 14260, USA \\ ${ }^{3}$ School of Economic Management, Hunan Institute of Technology, Hengyang 421001, China \\ ${ }^{4}$ International Business School, Jinan University, Zhuhai, Guangdong 519070, China
}

Correspondence should be addressed to Hao Guo; haoguo8701@gmail.com and Ying Zhang; yz29@buffalo.edu

Received 29 December 2017; Revised 26 September 2018; Accepted 15 October 2018; Published 24 October 2018

Academic Editor: Laszlo T. Koczy

Copyright (C) 2018 Congdong Li et al. This is an open access article distributed under the Creative Commons Attribution License, which permits unrestricted use, distribution, and reproduction in any medium, provided the original work is properly cited.

Customer returns are a common phenomenon in many industries, and they have a significant impact on business organizations and their supply chains. False failure returns are returned products that have no functional or cosmetic defects, and they represent a large body of customer returns in practice. In this paper, we develop a mixed-integer nonlinear programming model to study a multicommodity location-inventory problem in a forward-reverse logistics network. This model minimizes the total cost in this network by considering false failure returns, and it also considers many real-world business scenarios in forward and reverse logistics flows. Moreover, we design a new heuristic approach to solve the model efficiently. Finally, numerical experiments are conducted to validate our solution approach and provide meaningful managerial insights.

\section{Introduction}

Reverse logistics is an emerging topic that has attracted many research attentions because of the great importance of customer returns in today's business. Nowadays, the percentage of customer returns in total sales varies from 5\% to $9 \%$ for durable goods, and it is up to $35 \%$ for fashion apparel products [1]. Since customer returns are more frequent in e-commerce, return rate in online shopping has exceeded $18 \%$ of total sales, and especially, it reaches $74 \%$ for fashion products [2]. In the retail industry, the value of customer products has exceeded $\$ 100$ billion per year in the United States [3]. Although many products are returned because of quality issues, a lot of items that have no defects can still be returned for various reasons such as installation difficulties, bad user experience, or price competition. Those items represent a large body of customer returns, and they are defined as "false failure returns" [4].

Forward-reverse logistics network consist of forward flows that are directed from suppliers to customers and reverse flows of returned products from customers to suppliers. Since network design can contribute to reducing $60 \%$ the cost of a firm [5], Supply chain network design is an important strategic decision in supply chain management, and it is a big challenge to business managers. For example, many big companies such as Kodak, Xerox, and General Motors have developed their reverse logistics systems and those efforts are very successful [6]. Currently, many business decisions are made jointly to improve the efficiency of business organizations and their supply chains. Location-inventory problem (LIP) [7] is such a problem that integrates strategical facility location decisions and tactical inventory management decisions, and it can be studied by incorporating many realworld business scenarios to improve the performance of a logistics system.

In this paper, we study a multicommodity locationinventory problem (MCLIP) in a forward-reverse logistics network in which the reverse flow consists of false failure returns. This problem is formulated as a mixed-integer nonlinear programming model whose objective function 
is to minimize the total cost in such a network, and it incorporates many business decisions and processes such as facility locations, return processing, and order fulfillment. More specifically, the solution to this problem will determine the number and locations of hybrid distribution-collection centers (HDCCs), how to use those HDCCs to fulfill orders and collect returns, and how to replenish inventories in those HDCCs. Due to the complexity of LIPs, we also design an improved differential evolution algorithm (IDE) to solve the MCLIP efficiently. Computational results are presented to validate the solution approach, and research implications and managerial insights are also discussed.

The rest of this paper is organized as follows: In Section 2, we review related research works in the literature. In Section 3, we describe the research problem and present the mathematical model. In Section 4, we propose a new heuristic approach to solve the problem efficiently. In Section 5, we show computational results and discuss managerial insights. Section 6 concludes the paper and provides directions for future research.

\section{Literature Review}

The design of logistics networks involves several core components such as facility location and inventory management. LIPs have been extensively studied because of their great importance in practice, and Farahani et al. [8] provide a comprehensive review of such works in the literature. In this section, we review the research works on LIPs from the perspective of forward and reverse logistics, respectively.

In the literature, most LIPs are studied under forward logistics networks. For example, Daskin et al. [7] study a LIP with the risk-pooling effect and design a Lagrangian relaxation algorithm as the solution approach. Shen et al. [9] use the same integer programming model as that in Daskin et al.[7] and convert it into a set-covering integer programming model and then solve it by column generation. Moreover, LIPs are studied by incorporating many business scenarios and processes. For example, Ozsen et al. [10] consider capacitated warehouses and the impact of multisourcing in addition to Shen et al. [9]. Amiri-Aref et al. [11] study multisourcing location-inventory problem with stochastic demand. Silva et al. [12] and $\mathrm{Qu}$ et al. [13] study the stochastic location-inventory problems under different replenishment policies. Mousavi et al. [14] study a seasonal multiple-product location allocation and inventory control problem in a planning horizon consisting of multiple periods. Since business environments are always dynamic, demand uncertainty [15-17] and different risks [18] are also considered.

It is emerging to study LIPs in the reverse logistics. Since reverse logistics is an important part of closed-loop supply chains (CLSCs), those works are usually conducted with CLSCs. For example, Diabat et al. [19] study a closedloop location-inventory problem by considering spare parts, Asl-Najafi et al. [20] study a dynamic closed-loop locationinventory problem under disruption risk, and Kaya et al. [21] and Guo et al. [22] extend such studies by incorporating

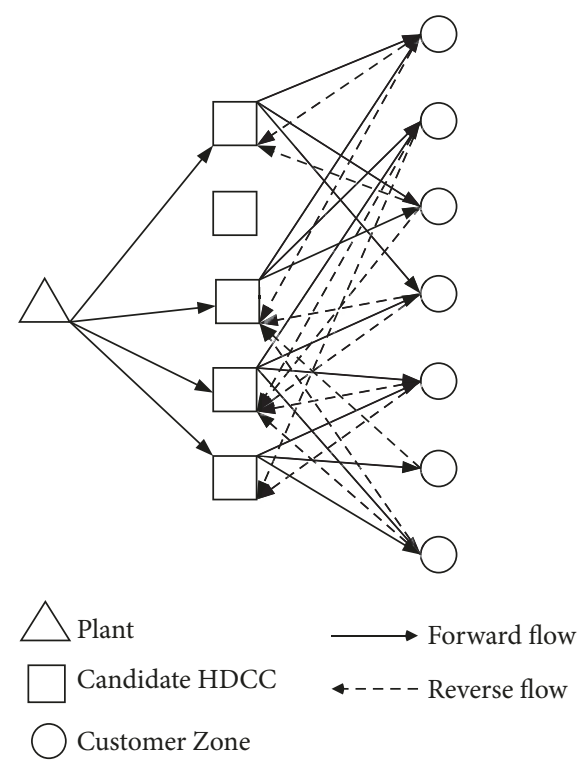

FIGURE 1: A forward-reverse logistics network with false failure returns.

pricing and vehicle routing decisions in CLSCs, respectively. Since returned products are usually sold to secondary markets in practice, LIPs are also studied by considering CLSCs and secondary markets $[23,24]$.

Although LIPs have been extensively studied from the perspective of reverse logistics, those works are still very limited because the types of customer returns are rarely considered. This paper studies a LIP in a forward-reverse logistics network in which the reverse logistics flow only consists of false failure returns. To the best of our knowledge, this is the first paper that considers false failure returns in the LIP literature, and it makes a significant contribution to the literature because of the great importance of false failure returns in practice.

\section{Mathematical Model}

3.1. Problem Description. We consider a three-echelon supply chain network in which a manufacturing plant ships multiple commodities to customer zones via a set of facilities. Moreover, returned products will be collected from customers and shipped to the same facilities. Therefore, those facilities are hybrid distribution-collection centers (HDCCs) [25, 26], and they have many advantages such as cost saving and pollution reduction by improving the efficiency of workflows. In this network, we assume that only false failure returns will be collected. Since those returns do not have any quality issues, they will not be sent to the plant for repair, and instead, they will be resold in the market after some simple processes (e.g., inspection, repackaging, etc.) in HDCCs. Figure 1 shows the forward-reverse logistics network under study. In this network, forward flows will be unidirectional from the plant to HDCCs and then from HDCCs to customer zones, but reverse flows will only be directed from customer zones to 
HDCCs because false failure returns will not be sent to the plant for repair.

This paper studies a multicommodity location-inventory problem (MCLIP) in the network shown in Figure 1, and we make the following assumptions to simplify the problem: (i) customer zones are predetermined and fixed; (ii) the returned products will be the same as new items when they are resold to the market; (iii) the distances between HDCCs and customer zones are Euclidean distances. In practice, this problem can be solved to answer the following questions:

(1) What are the best locations for HDCCs in this network?

(2) How to assign those HDCCs to customer zones in the forward and reverse networks, respectively?

(3) How to optimize inventory replenishment decisions at HDCCs?

\subsection{Notations}

Sets

$W$ : set of commodities, indexed by $w$;

$R$ : set of candidate HDCC locations, indexed by $r$;

$I$ : set of customer zones, indexed by $i$.

\section{Parameters}

$a_{r}$ : fixed (yearly) cost of building a HDCC at location $r$, for each $r \in R$;

$b_{r w}$ : fixed administrative and handling cost of placing an order for product $w$ at HDCC $r$, for each $r \in R$ and $w \in W$;

$c_{r w}$ : basic transportation cost per shipment of product $w$ from the plant to HDCC $r$, for each $r \in R$ and $w \in W$;

$e_{r w}$ : additional transportation cost per unit of product $w$ from the plant to HDCC $r$, for each $r \in R$ and $w \in W$; $d_{\text {riw }}$ : shipping cost per unit of product $w$ from HDCC $r$ to customer zone $i$, for each $r \in R, i \in I$ and $w \in W$;

$f_{r w}$ : repackaging/refurbishing cost per unit of returned product $w$ at HDCC $r$, for each $r \in R$ and $w \in W$;

$g_{r w}$ : inspection cost per unit of returned product $w$ at HDCC $r$, for each $r \in R$ and $w \in W$;

$h_{r w}$ : (yearly) holding cost per unit of product $w$ at HDCC $r$, for each $r \in R$ and $w \in W$;

$q_{w}$ : return rate of product $w$, for each $w \in W$;

$\mu_{i w}$ : mean (daily) demand of product $w$ at customer zone $i$, for each $i \in I$ and $w \in W$;

$\sigma_{i w}^{2}$ : variance of (daily) demand of product $w$ at customer zone $i$, for each $i \in I$ and $w \in W$;

$\alpha$ : desired percentage of market demand satisfied;

$z_{\alpha}$ : standard normal deviation such that $P\left(z \leq z_{\alpha}\right)=$ $\alpha$

$L$ : order lead time in days at HDCCs; $\lambda$ : working days per year;

$D_{r w}$ : mean (yearly) demand of product $w$ at HDCC $r$, for each $r \in R$ and $w \in W$.

\section{Decision Variables}

$Q_{r w}$ : order quantity of product $w$ per order at HDCC $r$;

$X_{r}=1$ if opening one HDCC at location $r$, and 0 otherwise, for each $r \in R$;

$Y_{\text {riw }}=1$ if HDCC $r$ fulfills demand at customer zone $i$, and 0 otherwise, for each $r \in R, i \in I$ and $w \in W$;

$Z_{\text {irw }}=1$ if HDCC $r$ collects returns from customer zone $i$, and 0 otherwise, for each $r \in R, i \in I$ and $w \in W$.

3.3. The Model. In this paper, the research problem is formulated by a mixed-integer nonlinear programming model. The objective of this model is to minimize the total cost in the forward-reverse logistics network, and it comprises (i) location costs, (ii) shipping costs from HDCCs to customer zones, (iii) inventory costs including working inventory and safety stock costs, and (iv) return costs.

3.3.1. Location Cost. Obviously, the total location cost $\left(C_{L}\right)$ can be written as

$$
C_{L}=\sum_{r \in R} a_{r} X_{r}
$$

3.3.2. Shipping Cost from HDCCs to Customer Zones. The total shipping cost $\left(C_{T}\right)$ from HDCCs to customer zones in the forward logistics flow can be expressed as follows:

$$
C_{T}=\lambda \sum_{r \in R} \sum_{w \in W} \sum_{i \in I} \mu_{i w} d_{r i w} Y_{r i w}
$$

3.3.3. Inventory Cost. Inventory strategy plays an important role in supply chain management. In this study, we assume that HDCCs order new products from the plant by using an $(R, Q)$ policy with type I service, and the total inventory cost includes working inventory and safety stock costs. The $(R, Q)$ model can be approximated by an economic order quantity (EOQ) model [9], and Axsater [27] shows that the maximum relative error introduced by using the EOQ model instead of the $(Q, r)$ policy is 0.118 . Therefore, we use an EOQ model to optimize the inventory strategy in this study, and the order frequency and quantity at a HDCC will be calculated by using the total demand of the customer zones served by this HDCC.

In general, the working inventory cost includes (1) fixed cost of placing orders, (2) shipping cost from the plant to HDCCs, and (3) holding cost of the working inventory. The individual cost terms are formulated as follows.

(1) Order Cost. The annual fixed cost of placing orders for product $w$ from HDCC $r$ to the plant is given by $b_{r w}\left(D_{r w} / Q_{r w}\right)$. 
(2) Shipping Cost from the Plant to HDCCs. Since false failure returns will be resold to the market after they are processed in HDCCs, shipping quantities from the plant to HDCCs will equal the difference between market demands and return volumes. Therefore, the total shipping cost from the plant to HDCC $r$ per year is given by

$$
c_{r w} \frac{D_{r w}}{Q_{r w}}+\lambda e_{r w}\left(\sum_{i \in I} \mu_{i w} Y_{r i w}-\sum_{i \in I} q_{w} \mu_{i w} Z_{i r w}\right) .
$$

(3) Holding Cost of the Working Inventory. In this study, the working inventory comes from two sources which are the new products ordered from the plant and the returned products collected from customer zones. Therefore, the forward and reverse logistics flows will be integrated at HDCCs, and the average inventory of product $w$ at HDCC $r$ during period $T$ can be expressed as follows:

$$
\begin{gathered}
\frac{1}{T} \int_{0}^{T}\left(S_{r w}-\frac{S_{r w}-q_{w} S_{r w}}{T}\right) d t=S_{r w}-\frac{1}{2} \theta_{r w} T \\
\quad=\frac{Q_{r w}}{1-q_{w}}-\frac{\left(1-q_{w}\right)}{2} S_{r w}=\frac{\left(1+q_{w}\right)}{2\left(1-q_{w}\right)} Q_{r w}
\end{gathered}
$$

where $S_{r w}$ is the mean daily demand of product $w$ at HDCC $r$. Consequently, the holding cost of the working inventory is given by

$$
\sum_{i \in I} \frac{\left(1+q_{w}\right) h_{r w}}{2\left(1-q_{w}\right)} Q_{r w}
$$

Using Eppen's risk-pooling result [28], the amount of safety stock to ensure that stockouts occur with a probability of $\alpha$ or less is $z_{\alpha} \sqrt{L \sum_{i \in I} \sigma_{i w}^{2} Y_{\text {riw }}}$, and the safety stock cost at HDCC $r$ is given by $h_{r w} z_{\alpha} \sqrt{L \sum_{i \in I} \sigma_{i w}^{2} Y_{r i w}}$.

Therefore, the total annual inventory cost $\left(C_{I}\right)$ of product $w$ at HDCC $r$ is given by

$$
\begin{aligned}
C_{I}= & b_{r w} \frac{D_{r w}}{Q_{r w}}+c_{r w} \frac{D_{r w}}{Q_{r w}} \\
& +\lambda e_{r w}\left(\sum_{i \in I} \mu_{i w} Y_{r i w}-\sum_{i \in I} q_{w} \mu_{i w} Z_{i r w}\right) \\
& +\sum_{i \in I} \frac{\left(1+q_{w}\right) h_{r w}}{2\left(1-q_{w}\right)} Q_{r w}+h_{r w} z_{\alpha} \sqrt{L \sum_{i \in I} \sigma_{i w}^{2} Y_{r i w}} .
\end{aligned}
$$

3.3.4. Return Cost. Returns costs are incurred by customer returns, and they include inspection cost, repackaging cost, and shipping cost from customer zones to HDCCs.

(1) Inspection Cost. Returned products will be inspected at HDCCs, and the total inspection cost is given by $\lambda \sum_{r \in R} \sum_{w \in W} \sum_{i \in I} q_{w} \mu_{i w} g_{r w} Z_{r i w}$.

(2) Repackaging Cost. The returned products also need to be repackaged before they are resold to the market, and the total repackaging cost is given by $\lambda \sum_{r \in R} \sum_{w \in W} \sum_{i \in I} q_{w} \mu_{i w} f_{r w} Z_{r i w}$.
(3) Shipping Cost from Customer Zones to HDCCs. The total shipping cost from customer zones to HDCCs is given by $\lambda \sum_{r \in R} \sum_{w \in W} \sum_{i \in I} q_{w} \mu_{i w} d_{r i w} Z_{r i w}$.

Therefore, the total annual return cost $\left(C_{R}\right)$ is given by

$$
C_{R}=\lambda \sum_{r \in R} \sum_{w \in W} \sum_{i \in I} q_{w} \mu_{i w}\left(g_{r w}+f_{r w}+d_{r i w}\right) Z_{r i w} .
$$

3.3.5. Optimization Model. Given the individual cost functions above, the research problem under study can be formulated by a mixed-integer nonlinear program as follows:

$$
\begin{aligned}
& \min Z=C_{L}+C_{T}+C_{I}+C_{R}=\sum_{r \in R} a_{r} X_{r} \\
& +\lambda \sum_{r \in R} \sum_{w \in W} \sum_{i \in I} \mu_{i w} d_{r i w} Y_{r i w}+\sum_{r \in R} \sum_{w \in W}\left\{b_{r w} \frac{D_{r w}}{Q_{r w}}\right. \\
& +c_{r w} \frac{D_{r w}}{Q_{r w}}+\lambda e_{r w}\left(\sum_{i \in I} \mu_{i w} Y_{r i w}-\sum_{i \in I} q_{w} \mu_{i w} Z_{i r w}\right) \\
& \left.+\sum_{i \in I} \frac{\left(1+q_{w}\right) h_{r w}}{2\left(1-q_{w}\right)} Q_{r w}+h_{r w} z_{\alpha} \sqrt{L \sum_{i \in I} \sigma_{i w}^{2} Y_{r i w}}\right\} \\
& +\lambda \sum_{r \in R} \sum_{w \in W} \sum_{i \in I} q_{w} \mu_{i w}\left(g_{r w}+f_{r w}+d_{r i w}\right) Z_{r i w}
\end{aligned}
$$

subject to

$$
\begin{aligned}
& \sum_{r \in R} X_{r} \geq 1 ; \\
& \sum_{r \in R} Y_{\text {riw }}=1, \quad \forall i \in I, \forall w \in W ; \\
& \sum_{r \in R} Z_{\text {irw }}=1, \quad \forall i \in I, \forall w \in W ; \\
& \sum_{r \in R} \sum_{w \in W} Y_{\text {riw }}=|W|, \quad \forall i \in I ; \\
& \sum_{r \in R} \sum_{w \in W} Z_{\text {irw }}=|W|, \quad \forall i \in I ; \\
& Y_{\text {riw }} \leq X_{r}, \quad \forall i \in I, \quad \forall r \in R, \forall w \in W ; \\
& Z_{\text {irw }} \leq X_{r}, \quad \forall i \in I, \quad \forall r \in R, \forall w \in W ; \\
& \sum_{w \in W} \sum_{i \in I} Y_{\text {riw }} \geq X_{r}, \quad \forall r \in R ; \\
& \sum_{i \in I} Z_{i r w} \leq M \sum_{i \in I} Y_{\text {riw }}, \quad \forall r \in R, \forall w \in W ; \\
& X_{r}=\{0,1\}, \quad \forall r \in R ; \\
& Y_{\text {riw }}=\{0,1\}, \quad \forall i \in I, \forall r \in R, \forall w \in W ; \\
& Z_{\text {irw }}=\{0,1\}, \quad \forall i \in I, \forall r \in R, \forall w \in W ;
\end{aligned}
$$

In this model, objective function (8) means that the total cost will be minimized. Constraint (9) means that at least one 
HDCC will be built. Constraint (10) means that the demand of a product in a customer zone will be fulfilled by only one HDCC. Constraint (11) means that the returns of a product from a customer zone will be collected by only one HDCC. Constraint (12) means that all types of products will be provided to a customer zone by HDCCs in the forward flow. Constraint (13) means that all types of returned products will be collected from a customer zone by HDCCs in the reverse flow. Constraints (14) and (15) mean that a customer zone can be assigned to a HDCC in the forward and reverse logistics flows, respectively, only if this HDCC is built. Constraint (16) means that a HDCC will serve at least one customer zone in the forward logistics network after it is built. Constraint (17) means that a HDCC will collect the returns of a product in the reverse flow only if it fulfills the demand of this product in the forward flow. Constraints (18)-(20) indicate that $X_{r}, Y_{\text {riw }}$, and $Z_{\text {irw }}$ are binary variables.

Note that decision variable $Q_{r w}$ only appears in the objective function. Since the objective function is convex in $Q_{r w}$, its optimal value can be obtained by taking the partial derivative of the objective function with respect to it, which gives

$$
Q_{r w}^{*}=\sqrt{\sum_{i \in I} \frac{2\left(1-q_{w}\right)\left(b_{r w}+c_{r w}\right) D_{r w}}{\left(1+q_{w}\right) h_{r w}}} .
$$

Therefore, the objective function can be rewritten as follows:

$$
\begin{aligned}
& \min Z=\lambda \sum_{r \in R} \sum_{w \in W} \sum_{i \in I} \mu_{i w} d_{r i w} Y_{\text {riw }} \\
& +\sum_{r \in R} \sum_{w \in W} \sqrt{\sum_{i \in I} \frac{2 \lambda h_{r w}\left(1+q_{w}\right)\left(b_{r w}+c_{r w}\right) \mu_{i w} Y_{r i w}}{\left(1-q_{w}\right)}} \\
& +\lambda \sum_{r \in R} \sum_{w \in W} \sum_{i \in I} e_{r w}\left(\mu_{i w} Y_{r i w}-q_{w} \mu_{i w} Z_{i r w}\right) \\
& +\sum_{r \in R} \sum_{w \in W} h_{r w} z_{\alpha} \sqrt{L \sum_{i \in I} \sigma_{i w}^{2} Y_{r i w}}+\sum_{r \in R} a_{r} X_{r} \\
& +\lambda \sum_{r \in R} \sum_{w \in W} \sum_{i \in I} q_{w} \mu_{i w}\left(g_{r w}+f_{r w}+d_{r i w}\right) Z_{r i w}
\end{aligned}
$$

\section{Solution Approach}

Location problems are NP-hard in general [29], and LIPs can be more complicated. Evolutionary algorithms are widely used to solve LIPs, and differential evolution (DE), which was first introduced by Storn and Price [30], is popular method to solve nonlinear and nonconvex functions [31]. Although DE has a strong global search ability to solve such problems, its performance is not always guaranteed due to its weaknesses such as the poor local search ability. To obtain a more stable and effective approach, we design an improved differential evolution algorithm (IDE) to solve MCLIP. More specifically, IDE enhances the performance of DE by two ways: First, IDE introduces opposition-based learning (OBL) [32] to generate better initial populations. Second, it uses a novel mutation
TABLE 1: IDE notations.

\begin{tabular}{ll}
\hline Notation & Explanation \\
\hline$N p$ & $\begin{array}{l}\text { population size } \\
\text { dimension (i.e., number of customer zones in this } \\
\text { study) } \\
\text { maximum number of generations for evolution }\end{array}$ \\
$G$ & $\begin{array}{l}\text { number of consecutive iterations if no better } \\
\text { individual }\end{array}$ \\
$K$ & $\begin{array}{l}\text { solution vector of individuals } i \text { in generation } g \\
(i=1,2, \ldots, N p)\end{array}$ \\
$Z^{i, g}$ & $\begin{array}{l}\text { mutant vector of individuals } i \text { in generation } g \\
(i=1,2, \ldots, N p)\end{array}$ \\
$V Z^{i, g}$ & $\begin{array}{l}\text { trial vector of individuals } i \text { in generation } g(i=1,2, \\
U Z^{i, g}\end{array}$ \\
$F^{i, g}$ & $\begin{array}{l}\text { mutation factor of individuals } i \text { in generation } g \\
(i=1,2, \ldots, N p)\end{array}$ \\
$C R$ & crossover factor \\
\hline
\end{tabular}

factor $F$ based on the Gaussian distribution $N(0,1)$ to enhance the diversity of populations.

4.1. Individuals and Operators. Generally, a DE algorithm has four main operations: initialization, mutation, crossover, and selection. After an initial population is created in the initialization step, the algorithm will execute mutation, crossover, and selection operations iteratively to improve the population until a stop criterion is satisfied. IDE follows the same procedure, and it uses notations shown in Table 1.

4.1.1. Initialization. When DE is applied to solve optimization problems, an encoding-decoding scheme will be needed to convert individuals in $\mathrm{DE}$ to the solutions to the optimization model, and vice versa. In IDE, an individual is represented by the matrix shown in (23).

$$
\begin{array}{r}
Z^{i, g} \\
=\left(\begin{array}{c}
x_{1,1}^{i, g}, x_{1,2}^{i, g}, \ldots, x_{1, N}^{i, g}, y_{1, N+1}^{i, g}, y_{1, N+2}^{i, g}, \ldots, y_{1,2 N}^{i, g} \\
\ldots \\
x_{W, 1}^{i, g}, x_{W, 2}^{i, g}, \ldots, x_{W, N}^{i, g}, y_{W, N+1}^{i, g}, y_{W, N+2}^{i, g}, \ldots, y_{W, 2 N}^{i, g}
\end{array}\right), \\
i=1,2, \ldots, N p ; g=1,2, \ldots, G ;
\end{array}
$$

This matrix has $W$ rows and $2 N$ columns. For the research problem under study, $W$ and $N$ denote the numbers of commodities and customers, respectively, and the first and last $N$ columns are related to the forward and reverse flows, respectively. More specifically, if $x_{w, j}^{i, g}=r$, then the demand of product $w$ in customer zone $j$ will be fulfilled by HDCC $r$ in the forward flow, and if $y_{w, j}^{i, g}=r$, then the returns of product $w$ from customer zone $j$ will be collected by HDCC $r$ in the reverse flow.

Figure 2 shows an example matrix for a MCLIP that has two commodities, ten customer zones, and five candidate HDCC locations. For product 1, HDCC 1 fulfills demands in customer zones 1,6 , and 10 and collects returns from 


\begin{tabular}{|c|c|c|c|c|c|c|c|c|c|c|c|c|c|c|c|c|c|c|c|c|}
\hline & \multicolumn{10}{|c|}{ Forward logistics } & \multicolumn{10}{|c|}{ Reverse logistics } \\
\hline Product & 1 & 2 & 3 & 4 & 5 & 6 & 7 & 8 & 9 & 10 & 1 & 2 & 3 & 4 & 5 & 6 & 7 & 8 & 9 & 10 \\
\hline Product 1 & 1 & 3 & 3 & 2 & 3 & 1 & 2 & 3 & 5 & 1 & 1 & 2 & 5 & 3 & 3 & 2 & 1 & 3 & 1 & 5 \\
\hline Product 2 & 2 & 2 & 3 & 5 & 3 & 2 & 3 & 5 & 2 & 5 & 3 & 2 & 2 & 3 & 2 & 5 & 5 & 2 & 5 & 3 \\
\hline
\end{tabular}

Figure 2: An example individual.

customer zones 1, 7, and 9; HDCC 2 fulfills demands in customer zones 4 and 7 and collects returns from customer zones 2 and 6; HDCC 3 fulfills demands in customer zones $2,3,5$, and 8 and collects returns from customer zones 4, 5, and 8; HDCC 5 fulfills the demand in customer zone 9 and collects returns from customer zones 3 and 10. For product 2 , location decisions can be also explained in the same manner.

The aim of encoding is transforming the initial individual to practical solution of LIP. The formulation of decoding is as follows.

To decode an individual to a solution to the optimization problem, we can use a mechanism shown in (24) and (25).

$$
\begin{aligned}
& x_{w, j}^{i, 0}=\operatorname{round}\left(x^{L}+\operatorname{rand} \cdot\left(x^{U}-x^{L}\right)\right) ; \\
& \quad i=1,2, \ldots, N p ; j=1,2, \ldots, N ; w=1,2, \ldots, W ; \\
& y_{w, j}^{i, 0}=U\left(\operatorname{round}\left(y^{L}+\operatorname{rand} \cdot(\text { length }(U)-1)\right)\right) ; \\
& i=1,2, \ldots, N p ; j=N+1, N+2, \ldots, 2 N ; w=1,2, \ldots, W ;
\end{aligned}
$$

where $U=\operatorname{unique}\left(x_{w, 1}^{i, g}, x_{w, 2}^{i, g}, \ldots, x_{w, N}^{i, g}\right)$ are distinct HDCCs that provide product $w$ in the forward flow, rand is a random variable that is uniformly distributed on $[0,1], x^{L}$ and $x^{U}$

$$
\overline{Z^{i, 0}}=\left(\begin{array}{l}
\overline{x_{1,1}^{i, 0}}, \overline{x_{1,2}^{i, 0}}, \ldots, \overline{x_{1, N}^{i, 0}}, \overline{y_{1, N+1}^{i, 0}}, \overline{y_{1, N+2}^{i, 0}}, \ldots, \overline{y_{1,2 N}^{i, 0}} \\
\overline{x_{W, 1}^{i, 0}}, \frac{\ldots}{x_{W, 2}^{i, 0}}, \ldots, \overline{x_{W, N}^{i, 0}}, \frac{\cdots}{y_{W, N+1}^{i, 0}}, \overline{y_{W, N+2}^{i, 0}}, \ldots, \overline{y_{W, 2 N}^{i, 0}}
\end{array}\right)
$$

where $\overline{x_{w, j}^{i, 0}}$ is the entry in row $w$ and column $j$ of the $i^{\text {th }}$ individual in the opposite population and $\overline{y_{w, j}^{i, 0}}(i=1,2, \ldots, N p$; $j=N+1, N+2, \ldots, 2 N ; w=1,2, \ldots, W)$ is calculated by $(25)$.

Step 3. Select the $N p$ best solutions from $\left\{Z^{i, 0} \cup \overline{Z^{i, 0}}\right\}$ as initial population.

4.1.2. Mutation. The mutation strategy plays an important role $\mathrm{DE}$ to enhance the global optimization ability and convergence speed. Usually, DE will use DE/rand/1/bin as the mutation operation that will produce a new vector by adding the weighted difference of two randomly selected vectors are the lower and upper bounds of $x_{w, j}^{i, 0}$, and round is the rounding function. Obviously, we have $x^{L}=y^{L}=1$, and $x^{U}$ is decided by the number of candidate HDCC locations. The expression of $y_{w, j}^{i, 0}$ ensures that product $w$ can be returned to a HDCC in the reverse flow only if it provides this product in the forward flow, which is reinforced by Constraint (17) in the optimization model shown in Section 3.3.5.

IDE introduce a new mechanism based on OBL to generate better initial populations. More specifically, IDE will improve an initial population that is generated randomly by checking its opposite population by the following steps.

Step 1. Generate an initial population $Z^{i, 0}$ randomly:

$$
\begin{array}{r}
Z^{i, 0} \\
=\left(\begin{array}{c}
x_{1,1}^{i, 0}, x_{1,2}^{i, 0}, \ldots, x_{1, N}^{i, 0}, y_{1, N+1}^{i, 0}, y_{1, N+2}^{i, 0}, \ldots, y_{1,2 N}^{i, 0} \\
\ldots \\
x_{W, 1}^{i, 0}, x_{W, 2}^{i, 0}, \ldots, x_{W, N}^{i, 0}, y_{W, N+1}^{i, 0}, y_{W, N+2}^{i, 0}, \ldots, y_{W, 2 N}^{i, 0}
\end{array}\right), \\
i=1,2, \ldots, N p ;
\end{array}
$$

Step 2. Obtain its opposite population $\overline{Z^{i, 0}}$ : to the third vector in generation $g$ [30]. In this study, we design a new mutation strategy to improve the diversity of populations, and new mutant vectors $v x_{w, j}^{i, g+1}$ and $v y_{w, j}^{i, g+1}$ are generated by (28) and (29).

$$
\begin{aligned}
& v x_{w, j}^{i, g+1}=\operatorname{round}\left(x_{w, j}^{r 1, g}+F^{i, g} \cdot\left(x_{w, j}^{r 2, g}-x_{w, j}^{r 3, g}\right)\right) ; \\
& \quad i=1,2, \ldots, N p ; j=1,2, \ldots N ; w=1,2, \ldots, W ; \\
& v y_{w, j}^{i, g+1}=\operatorname{round}\left(y_{w, j}^{r 1, g}+F^{i, g} \cdot\left(y_{w, j}^{r 2, g}-y_{w, j}^{r 3, g}\right)\right) ; \\
& i=1,2, \ldots, N p ; j=N+1, N+2, \ldots, 2 N ; w=1,2, \ldots, W ;
\end{aligned}
$$

where $i \neq r 1 \neq r 2 \neq r 3, r 1, r 2, r 3$ are randomly selected from $\{1,2, \ldots, N p\}, F^{i, g}=F \cdot \xi^{i, g}$ and it amplifies the difference 
between two vectors, $F$ is an initial mutation factor, and $\xi^{i, g}$ is independently generated from the normal distribution $N(0,1)$. In the mutation step, the mutation factor will be updated first and then used to generate new mutant vectors, which will improve local and global search abilities and generate better mutant vectors. If $v x_{w, j}^{i, g+1}$ and $v y_{w, j}^{i, g+1}$ in a new mutant vector exceed the upper and lower bounds, then the vector will be generated again within its range.

4.1.3. Crossover. The crossover operation mixes mutate and target vectors to generate trial vectors and increase the diversity of populations. In IDE, the trial vector is generated by (30) and (31).

$$
\begin{aligned}
& u x_{w, j}^{i, g+1}= \begin{cases}v x_{w, j}^{i, g} & \operatorname{rand}(i)<C R \\
x_{w, j}^{i, g} & \text { otherwise }\end{cases} i=1,2, \ldots, N p ; j=1,2, \ldots, N ; w=1,2, \ldots, W ; \\
& u y_{w, j}^{i, g+1}= \begin{cases}v y_{w, j}^{i, g} & \operatorname{rand}(i)<C R \\
y_{w, j}^{i, g} & \text { otherwise }\end{cases} \\
& i=1,2, \ldots, N p ; j=N+1, N+2, \ldots, 2 N ; w=1,2, \ldots, W ;
\end{aligned}
$$

where $\operatorname{rand}(i)$ is random variable that is uniformly distributed on $[0,1]$, and $C R \in[0,1]$ is a predefined crossover rate.

4.1.4. Feasibility Correction. A new individual that is generated by mutation or crossover operations may not be feasible by violating Constraint (17). Therefore, a feasibility correction procedure is developed to exclude infeasible individuals. In this study, a new individual will be checked after it is generated. If Constraint (17) is violated, then it will be replaced by a new individual that is randomly generated by (25).

4.1.5. Selection. After a new population is generated, the selection operation will be executed to evaluate the objective values of all trial vectors. In IDE, a trial vector $f\left(U X^{i, g+1}\right)$ will be compared with the corresponding target vector $f\left(X^{i, g}\right)$ on their objective values by using a greedy criterion[30]. More specifically, the selection operation can be expressed as follows:

$$
Z^{i, g+1}= \begin{cases}U Z^{i, g+1} & f\left(U Z^{i, g+1}\right)<f\left(Z^{i, g}\right) \\ Z^{i, g} & \text { otherwise. }\end{cases}
$$

4.1.6. Stop Criterion. In DE, the mutation, crossover, and selection operations will be executed iteratively until a stop criterion is satisfied. In this study, IDE will stop until any of the criteria below is satisfied.

(1) No better solution is found in consecutive $K$ iterations.

(2) The maximum number of iterations, i.e., $G$, is reached.

4.2. Algorithm Flow. In summary, IDE consists of the following steps.
Step 1. Set $g=0$ and $k=0$.

Step 2. Create an initial population that consists of $N p$ individuals by applying OBL, and calculate the objective values of all individuals in the population.

Step 3. Execute mutation operation.

Step 4. Execute crossover operation.

Step 5. Perform feasibility correction.

Step 6. Execute selection operation.

Step 7. Set $g=g+1$. Calculate the objective values of all individuals in the new population. If the minimal objective value is less than that in the old population, set $k=1$. Otherwise, set $k=k+1$.

Step 8. If $k=K$ or $g=G$, stop the algorithm. Otherwise, go to Step 4.

\section{Computational Results}

In this section, numerical analysis is presented to validate IDE and provide managerial insights. First, we show how to obtain the optimal setting in IDE for the best possible performance. Second, the solution to an example MCLIP is presented. Third, sensitivity analysis is conducted to study the influences of the parameters in MCLIP. Last, IDE is compared with Lingo 11 and DE to validate its performance. All the experiments are implemented by Java JDK 1.7 on a Windows PC (AMD A10-9600P RADEON R5, 10 COMPUTE CORES 4C+6G 2.40GHz; RAM: 4.00 GB DDR; OS: Windows $10)$.

To get started, we use an example MCLIP that consists of eight candidate HDCC locations, eighty customer zones, and three commodities. The candidate HDCC locations and customer zones are uniformly distributed on a grid of $[0,50] \times[0,50]$, and the parameters are shown in Table 2 .

5.1. Parameter Analysis. The performance of DE can be significantly affected by its parameters or the combinations of those parameters. In this subsection, we test the performance of IDE under different $F$ and $C R$ values. In this experiment, we set $N p=4 S(S=N * W)$ and the algorithm was executed 30 times under each setting, and the numerical results are shown in Table 3.

From Table 3, we can see that $F$ and $C R$ have a significant impact on the searching ability and convergence speed of IDE, which are indicated by OS ratio and CPU time, respectively. Obviously, IDE has the best performance when $F=0.1$ and $C R=0.1$, and this setting will be used in the subsequent experiments.

5.2. Illustrative Example. This subsection shows the optimal to the example MCLIP. Table 4 shows the optimal solution which indicates HDCC locations and the corresponding customer zones in the forward and reverse flows, and Table 5 
TABLE 2: MCLIP parameters.

\begin{tabular}{lccccc}
\hline Parameters & Value & Parameters & Value & Parameters & Value \\
\hline$a_{r}$ & $U[1000,1500]$ & $b_{r w}$ & $U[5,10]$ & $c_{r w}$ & 10 \\
$e_{r w}$ & 5 & $f_{r w}$ & $U[1,3]$ & $g_{r w}$ & $U[1,3]$ \\
$h_{r w}$ & $U[1,3]$ & $q_{w}$ & $U[0.3,0.6]$ & $\mu_{i w}$ & $U[20,30]$ \\
$\sigma_{i w}{ }^{2}$ & $\sigma_{i}{ }^{2}=\mu_{i}$ & $z_{\alpha}$ & 1.96 & $L$ & 1 \\
\hline
\end{tabular}

Remark: $U[\mathrm{a}, \mathrm{b}]$ denotes the uniform distribution over $[\mathrm{a}, \mathrm{b}]$.

TABLE 3: IDE parameter test results.

\begin{tabular}{|c|c|c|c|c|c|c|c|c|c|c|}
\hline \multirow{2}{*}{\multicolumn{2}{|c|}{$F$}} & \multicolumn{9}{|c|}{$C R$} \\
\hline & & 0.1 & 0.2 & 0.3 & 0.4 & 0.5 & 0.6 & 0.7 & 0.8 & 0.9 \\
\hline OS Ratio & \multirow{2}{*}{0.1} & $96.67 \%$ & $56.67 \%$ & $36.67 \%$ & $3.33 \%$ & $0.00 \%$ & $0.00 \%$ & $0.00 \%$ & $0.00 \%$ & $0.00 \%$ \\
\hline CPU Time & & 28.42 & 22.74 & 20.13 & 18.90 & 17.79 & 17.16 & 17.15 & 16.78 & 17.33 \\
\hline OS Ratio & \multirow{2}{*}{0.2} & $96.67 \%$ & $83.33 \%$ & $23.33 \%$ & $10.00 \%$ & $3.33 \%$ & $0.00 \%$ & $0.00 \%$ & $0.00 \%$ & $0.00 \%$ \\
\hline CPU Time & & 42.01 & 35.09 & 31.17 & 29.26 & 27.49 & 26.76 & 26.41 & 26.22 & 27.18 \\
\hline OS Ratio & \multirow{2}{*}{0.3} & $0.00 \%$ & $56.67 \%$ & $50.00 \%$ & $20.00 \%$ & $3.33 \%$ & $0.00 \%$ & $0.00 \%$ & $0.00 \%$ & $0.00 \%$ \\
\hline CPU Time & & 47.40 & 46.46 & 43.12 & 40.61 & 38.78 & 37.51 & 36.65 & 36.72 & 37.19 \\
\hline OS Ratio & \multirow{2}{*}{0.4} & $0.00 \%$ & $0.00 \%$ & $0.00 \%$ & $3.33 \%$ & $6.67 \%$ & $0.00 \%$ & $0.00 \%$ & $0.00 \%$ & $0.00 \%$ \\
\hline CPU Time & & 46.89 & 47.66 & 46.78 & 46.04 & 45.43 & 44.88 & 44.63 & 44.37 & 44.32 \\
\hline OS Ratio & \multirow{2}{*}{0.5} & $0.00 \%$ & $0.00 \%$ & $0.00 \%$ & $0.00 \%$ & $0.00 \%$ & $0.00 \%$ & $0.00 \%$ & $0.00 \%$ & $0.00 \%$ \\
\hline CPU Time & & 47.01 & 47.00 & 45.49 & 46.61 & 46.18 & 46.28 & 46.08 & 44.13 & 45.53 \\
\hline OS Ratio & \multirow{2}{*}{0.6} & $0.00 \%$ & $0.00 \%$ & $0.00 \%$ & $0.00 \%$ & $0.00 \%$ & $0.00 \%$ & $0.00 \%$ & $0.00 \%$ & $0.00 \%$ \\
\hline CPU Time & & 45.89 & 47.56 & 47.17 & 46.26 & 44.70 & 46.78 & 47.01 & 45.96 & 44.89 \\
\hline OS Ratio & \multirow{2}{*}{0.7} & $0.00 \%$ & $0.00 \%$ & $0.00 \%$ & $0.00 \%$ & $0.00 \%$ & $0.00 \%$ & $0.00 \%$ & $0.00 \%$ & $0.00 \%$ \\
\hline CPU Time & & 47.45 & 47.03 & 46.94 & 47.53 & 44.71 & 45.39 & 46.70 & 45.14 & 45.11 \\
\hline OS Ratio & \multirow{2}{*}{0.8} & $0.00 \%$ & $0.00 \%$ & $0.00 \%$ & $0.00 \%$ & $0.00 \%$ & $0.00 \%$ & $0.00 \%$ & $0.00 \%$ & $0.00 \%$ \\
\hline CPU Time & & 47.23 & 47.82 & 46.28 & 45.75 & 45.43 & 45.27 & 43.85 & 46.25 & 46.59 \\
\hline OS Ratio & \multirow{2}{*}{0.9} & $0.00 \%$ & $0.00 \%$ & $0.00 \%$ & $0.00 \%$ & $0.00 \%$ & $0.00 \%$ & $0.00 \%$ & $0.00 \%$ & $0.00 \%$ \\
\hline CPU Time & & 48.08 & 44.82 & 44.95 & 46.98 & 46.45 & 47.66 & 45.06 & 45.77 & 43.33 \\
\hline
\end{tabular}

Remark: in Table 3, the optimal solutions are obtained by Lingo 11, and "OS Ratio" means the percentage of finding optimal solutions by IDE.

shows the optimal value and individual costs in the optimal solution.

5.3. Sensitivity Analysis. The optimal solution of a MCLIP can be affected significantly by important business parameters. In this subsection, sensitivity analysis is conducted on $q_{w}$, $d_{r i w}, b_{r w}, c_{r w}, e_{r w}, h_{r w}, g_{r w}$, and $f_{r w}$ to study their influences. More specifically, a parameter will be tested by changing its value by $(-30 \%, 30 \%)$ and fixing the other parameters and the numerical results are shown in Table 6.

From Table 6, we can see that optimal values can change significantly due to the change of those parameters. For example, the optimal value will decrease by $6.63 \%$ if $q_{w}$ decreases by $30 \%$, and the optimal value will increase by $6.65 \%$ if $q_{w}$ increases by $30 \%$. The influences of the parameters on individual costs are summarized as follows: When $q_{w}$ increases, $C_{R}$ will increase, $C_{I}$ and will decrease, but $C_{L}$ and $C_{T}$ will not change. When $d_{\text {riw }}$ increases, $C_{T}$ and $C_{R}$ will increase but $C_{L}$ and $C_{I}$ will not change. When $b_{r w}, c_{r w}, e_{r w}, h_{r w}$ increase, only $C_{I}$ will increase and the other costs will not change. When $g_{r w}$ or $f_{r w}$ increases, only $C_{I}$ will increase. We can see that, in general, $d_{\text {riw }}$ and $q_{w}$ have the most significant influences. Moreover, the optimal value is more sensitive when $d_{r i w}, q_{w}$, and $e_{r w}$ are relatively small. In practice, business managers may focus more on those parameters to stabilize the supply chain performance.

5.4. Performance Analysis. In this subsection, IDE is compared with Lingo 11 and DE to validate its performance in terms of solution accuracy and CPU time efficiency, and the three approaches are tested on a set of small(i.e., $20 \times 5 \times 2 / 3 / 4,40 \times 6 \times 2 / 3 / 4$ ), medium- (i.e., $50 \times 5 / 6 / 7 \times 3$, $70 \times 6 / 7 / 8 \times 3$ ), and large-sized problems (i.e., $80 \times 8 \times 2 / 3 / 4$, $100 \times 8 / 9 / 10 \times 3)$. Since population size $N p$ is an important parameter in IDE and it may vary according to the problem sizes, its value needs to be set properly to get the best possible performance. According to Figures 3-5 (in which "red line" represents solution accuracy in terms of the percentage of finding the optimal solutions, and "blue line" represents the mean CPU time), IDE will have the best performance when $N p=3 S, 4 S$, $5 S$ for small-, medium-, and large-sized problems, respectively. Therefore, we will use these settings in the subsequent experiments. 
TABLE 4: Optimal solution to the example MCLIP.

\begin{tabular}{|c|c|c|c|}
\hline HDCC Location & Commodity & $\begin{array}{l}\text { Customer Zone } \\
\text { (Forward Flow) }\end{array}$ & $\begin{array}{l}\text { Customer Zone } \\
\text { (Reverse Flow) }\end{array}$ \\
\hline \multirow{3}{*}{1} & 1 & $48,64,75$ & $48,64,75$ \\
\hline & 2 & $48,64,75$ & $48,64,75$ \\
\hline & 3 & $48,64,75$ & $48,64,75$ \\
\hline \multirow{3}{*}{2} & 1 & $4,9.10,11,26,29,30,39,44,45,47,57,68,72$ & $4,9.10,11,26,29,30,39,44,45,47,57,68,72$ \\
\hline & 2 & $4,9.10,11,26,29,30,39,44,45,47,57,68,72$ & $4,9.10,11,26,29,30,39,44,45,47,57,68,72$ \\
\hline & 3 & $4,9.10,11,26,29,30,39,44,45,47,57,68,72$ & $4,9.10,11,12,26,29,30,39,44,45,47,57,68,71,72$ \\
\hline \multirow{3}{*}{3} & 1 & $12,14,18,22,32,34,43,60,70,71$ & $12,14,18,22,32,34,43,60,70,71$ \\
\hline & 2 & $12,14,18,22,32,34,43,60,70,71$ & $12,14,18,22,32,34,38,43,60,70,71,79$ \\
\hline & 3 & $12,14,18,22,32,34,43,60,70,71$ & $14,18,22,32,34,43,60,70$ \\
\hline \multirow{3}{*}{4} & 1 & $2,7,13,35,52,56,58,63,76,80$ & $2,7,35,52,56,58,80$ \\
\hline & 2 & $2,7,13,35,52,56,58,63,76,80$ & $2,7,13,35,52,56,58,63,76,80$ \\
\hline & 3 & $2,7,13,35,52,56,58,63,76,80$ & $2,7,8,13,21,35,51,52,56,58,61,63,76,80$ \\
\hline \multirow{3}{*}{5} & 1 & $5,15,16,19,31,37,38,42,49,62,77,79$ & $5,15,16,19,31,37,38,42,49,62,77,79$ \\
\hline & 2 & $5,15,16,19,31,37,38,42,49,62,77,79$ & $5,15,16,19,31,37,42,49,62,77$ \\
\hline & 3 & $5,15,16,19,31,37,38,42,49,62,77,79$ & $5,15,16,19,31,37,38,42,49,62,77,79$ \\
\hline \multirow{3}{*}{6} & 1 & $3,6,17,20,23,24,36,67,69$ & $3,17,20,23,24,36,67,69$ \\
\hline & 2 & $3,6,17,20,23,24,36,67,69$ & $3,6,17,20,23,24,36,67,69$ \\
\hline & 3 & $3,6,17,20,23,24,36,67,69$ & $3,6,17,20,23,24,36,67,69$ \\
\hline \multirow{3}{*}{7} & 1 & $8,21,25,28,46,51,53,54,61,65,73,74$ & $6,8,13,21,25,27,2846,51,53,54,61,63,65,73,74,76$ \\
\hline & 2 & $8,21,25,28,46,51,53,54,61,65,73,74$ & $8,21,25,28,46,51,53,54,61,65,73,74$ \\
\hline & 3 & $8,21,25,28,46,51,53,54,61,65,73,74$ & $25,28,46,53,54,65,73,74$ \\
\hline \multirow{3}{*}{8} & 1 & $1,27,33,40,41,50,55,59,66,78$ & $1,33,40,41,50,55,59,66,78$ \\
\hline & 2 & $1,27,33,40,41,50,55,59,66,78$ & $1,27,33,40,41,50,55,59,66,78$ \\
\hline & 3 & $1,27,33,40,41,50,55,59,66,78$ & $1,27,33,40,50,55,59,66,78$ \\
\hline
\end{tabular}

TABLE 5: Optimal value and individual costs.

\begin{tabular}{lcccc}
\hline$C_{L}$ & $C_{T}$ & $C_{I}$ & $C_{R}$ & Total \\
\hline 10262 & 16651085.12 & 4665316.68 & 11617106.42 & 32943770.22 \\
\hline
\end{tabular}

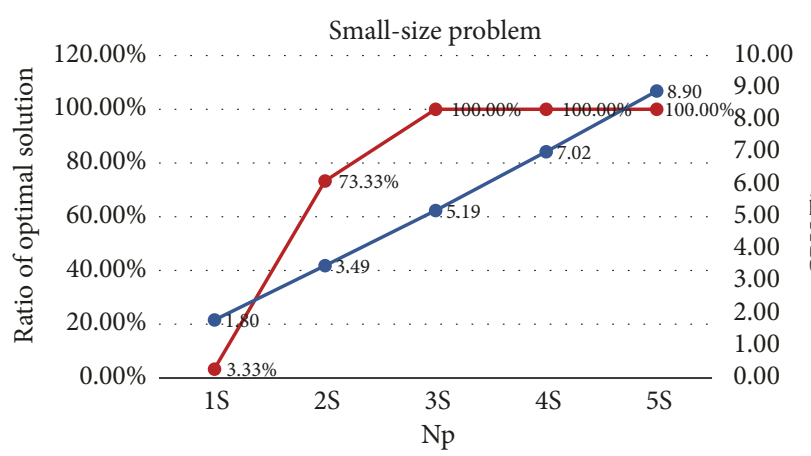

FIGURE 3: Analysis of $N p$ for small-sized problems $(40 \times 6 \times 4)$.

Table 7 shows the comparison results between IDE, DE, and Lingo 11. To avoid biased results, IDE and DE were executed 30 times for each test instance. Therefore, in this table, "O.V." means the optimal value, "O.V. (Best)" and "O.V. (Mean)" represent the best and mean optimal values in 30 runs, respectively, "OS Ratio" means the percentage of finding the optimal solutions, and "S.D." means the standard deviation of optimal values in 30 runs.

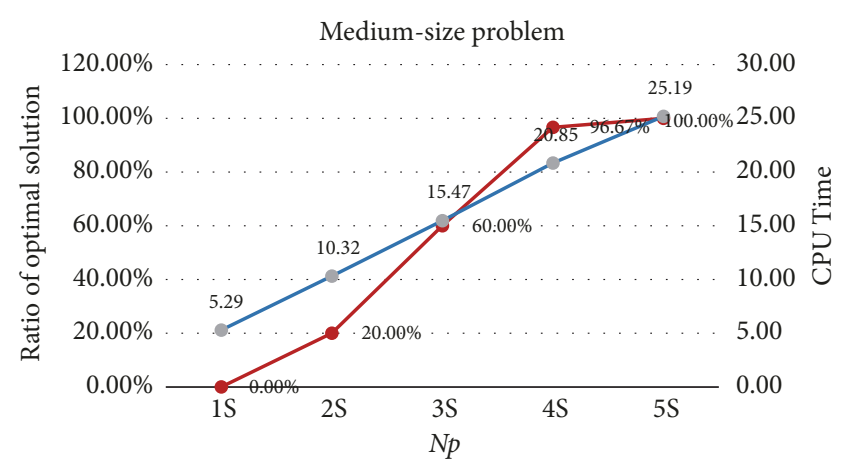

Figure 4: Analysis of $N p$ for medium-sized problems $(70 \times 8 \times 3)$.

From Table 7, we can see that IDE is the most effective and efficient method to solve MCLIPs, and its advantages over Lingo 11 and DE are summarized as follows:

(1) The optimal values obtained by IDE and Lingo 11 were almost identical, but IDE has better runtimes than Lingo 11. This indicates that IDE is much more efficient than Lingo 11 with the same solution accuracy. 
TABLE 6: Sensitivity analysis results.

\begin{tabular}{|c|c|c|c|c|c|c|c|c|}
\hline & $\Delta$ & $-30.00 \%$ & $-20.00 \%$ & $-10.00 \%$ & $0.00 \%$ & $10.00 \%$ & $20.00 \%$ & $30.00 \%$ \\
\hline \multirow{6}{*}{$q_{w}$} & $C_{I}$ & 5967659.67 & 5532973.87 & 5098809.85 & 4665316.678 & 4232715.54 & 3801356.65 & 3371846.74 \\
\hline & Gap & $27.92 \%$ & $18.60 \%$ & $9.29 \%$ & $0.00 \%$ & $-9.27 \%$ & $-18.52 \%$ & $-27.73 \%$ \\
\hline & $C_{R}$ & 8131974.50 & 9293685.14 & 10455395.78 & 11617106.42 & 12778817.06 & 13940527.71 & 15102238.35 \\
\hline & Gap & $-30.00 \%$ & $-20.00 \%$ & $-10.00 \%$ & $0.00 \%$ & $10.00 \%$ & $20.00 \%$ & $30.00 \%$ \\
\hline & $\mathrm{OV}$ & 30760981.29 & 31488006.13 & 32215552.76 & 32943770.22 & 33672879.73 & 34403231.48 & 35135432.21 \\
\hline & Gap & $-6.63 \%$ & $-4.42 \%$ & $-2.21 \%$ & $0.00 \%$ & $2.21 \%$ & $4.43 \%$ & $6.65 \%$ \\
\hline \multirow{6}{*}{$d_{r i w}$} & $C_{T}$ & 11655759.59 & 13320868.10 & 14985976.61 & 16651085.12 & 18316193.63 & 19981302.15 & 21646410.66 \\
\hline & Gap & $-30.00 \%$ & $-20.00 \%$ & $-10.00 \%$ & $0.00 \%$ & $10.00 \%$ & $20.00 \%$ & $30.00 \%$ \\
\hline & $C_{R}$ & 9141586.34 & 9969691.59 & 10793951.68 & 11617106.42 & 12437398.32 & 13255554.35 & 14073565.94 \\
\hline & Gap & $-21.31 \%$ & $-14.18 \%$ & $-7.09 \%$ & $0.00 \%$ & $7.06 \%$ & $14.10 \%$ & $21.15 \%$ \\
\hline & $\mathrm{OV}$ & 25472924.60 & 27966138.37 & 30455506.97 & 32943770.22 & 35429170.64 & 37912435.18 & 40395555.28 \\
\hline & Gap & $-22.68 \%$ & $-15.11 \%$ & $-7.55 \%$ & $0.00 \%$ & $7.54 \%$ & $15.08 \%$ & $22.62 \%$ \\
\hline \multirow{4}{*}{$b_{r w}$} & $C_{I}$ & 4659545.89 & 4661513.59 & 4663436.18 & 4665316.678 & 4667157.74 & 4668961.77 & 4670730.94 \\
\hline & Gap & $-0.12 \%$ & $-0.08 \%$ & $-0.04 \%$ & $0.00 \%$ & $0.04 \%$ & $0.08 \%$ & $0.12 \%$ \\
\hline & $\mathrm{OV}$ & 32937999.43 & 32939967.13 & 32941889.73 & 32943770.22 & 32945611.28 & 32947415.32 & 32949184.48 \\
\hline & Gap & $-0.02 \%$ & $-0.01 \%$ & $-0.01 \%$ & $0.00 \%$ & $0.01 \%$ & $0.01 \%$ & $0.02 \%$ \\
\hline \multirow{4}{*}{$c_{r w}$} & $C_{I}$ & 4657224.85 & 4660009.99 & 4662704.48 & 4665316.678 & 4667853.73 & 4670321.79 & 4672726.23 \\
\hline & Gap & $-0.17 \%$ & $-0.11 \%$ & $-0.06 \%$ & $0.00 \%$ & $0.05 \%$ & $0.11 \%$ & $0.16 \%$ \\
\hline & $\mathrm{OV}$ & 32935678.39 & 32938463.54 & 32941158.03 & 32943770.22 & 32946307.27 & 32948775.33 & 32951179.77 \\
\hline & Gap & $-0.02 \%$ & $-0.02 \%$ & $-0.01 \%$ & $0.00 \%$ & $0.01 \%$ & $0.02 \%$ & $0.02 \%$ \\
\hline \multirow{4}{*}{$e_{r w}$} & $C_{I}$ & 3292753.68 & 3750274.68 & 4207795.68 & 4665316.678 & 5122837.68 & 5580358.68 & 6037879.68 \\
\hline & Gap & $-29.42 \%$ & $-19.61 \%$ & $-9.81 \%$ & $0.00 \%$ & $9.81 \%$ & $19.61 \%$ & $29.42 \%$ \\
\hline & $\mathrm{OV}$ & 31571207.22 & 32028728.22 & 32486249.22 & 32943770.22 & 33401291.22 & 33858812.22 & 34316333.22 \\
\hline & Gap & $-4.17 \%$ & $-2.78 \%$ & $-1.39 \%$ & $0.00 \%$ & $1.39 \%$ & $2.78 \%$ & $4.17 \%$ \\
\hline \multirow{4}{*}{$h_{r w}$} & $C_{I}$ & 4650403.35 & 4655668.91 & 4660623.12 & 4665316.678 & 4669787.84 & 4674066.35 & 4678175.84 \\
\hline & Gap & $-0.32 \%$ & $-0.21 \%$ & $-0.10 \%$ & $0.00 \%$ & $0.10 \%$ & $0.19 \%$ & $0.28 \%$ \\
\hline & $\mathrm{OV}$ & 32928856.89 & 32934122.46 & 32939076.67 & 32943770.22 & 32948241.39 & 32952519.89 & 32956629.38 \\
\hline & Gap & $-0.05 \%$ & $-0.03 \%$ & $-0.01 \%$ & $0.00 \%$ & $0.01 \%$ & $0.03 \%$ & $0.04 \%$ \\
\hline \multirow{4}{*}{$g_{r w}$} & $C_{R}$ & 11058612.56 & 11245420.25 & 11431721.41 & 11617106.42 & 11802239.42 & 11987372.42 & 12172505.42 \\
\hline & Gap & $-4.81 \%$ & $-3.20 \%$ & $-1.60 \%$ & $0.00 \%$ & $1.59 \%$ & $3.19 \%$ & $4.78 \%$ \\
\hline & $\mathrm{OV}$ & 32385276.36 & 32572084.05 & 32758385.21 & 32943770.22 & 33128903.22 & 33314036.22 & 33499169.22 \\
\hline & Gap & $-1.70 \%$ & $-1.13 \%$ & $-0.56 \%$ & $0.00 \%$ & $0.56 \%$ & $1.12 \%$ & $1.69 \%$ \\
\hline \multirow{4}{*}{$f_{r w}$} & $C_{R}$ & 11146187.90 & 11303872.70 & 11461479.65 & 11617106.42 & 11770266.78 & 11922189.48 & 12073560.78 \\
\hline & Gap & $-4.05 \%$ & $-2.70 \%$ & $-1.34 \%$ & $0.00 \%$ & $1.32 \%$ & $2.63 \%$ & $3.93 \%$ \\
\hline & $\mathrm{OV}$ & 32472851.69 & 32630536.49 & 32788143.45 & 32943770.22 & 33096930.58 & 33248853.28 & 33400224.58 \\
\hline & Gap & $-1.43 \%$ & $-0.95 \%$ & $-0.47 \%$ & $0.00 \%$ & $0.46 \%$ & $0.93 \%$ & $1.39 \%$ \\
\hline
\end{tabular}

Remark: in Table 6, "OV" means the optimal value, and "Gap" means the change of the optimal value. Note that the gap will be zero if no parameter is changed.

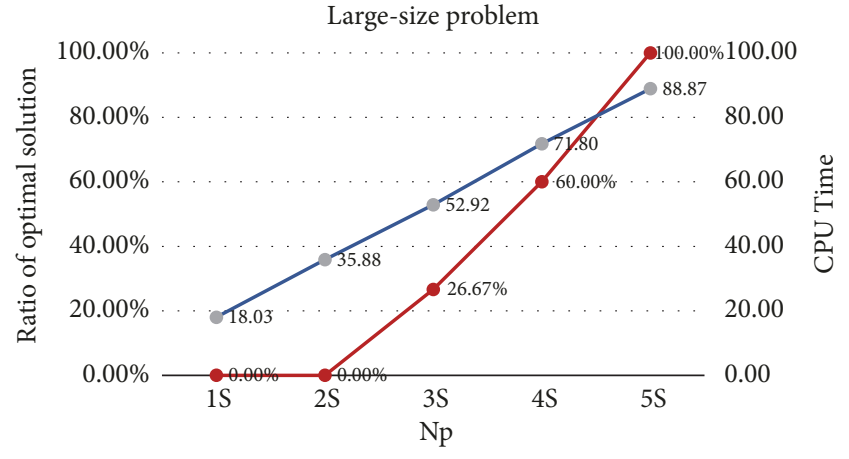

Figure 5: Analysis of $N p$ for large-sized problems $(100 \times 10 \times 3)$.
(2) IDE and DE can achieve similar solution accuracies on small- and medium-sized problems. However, the optimal values from DE are significantly larger than those from IDE on large-sized problems. Particularly, DE cannot find optimal solutions for the problems whose sizes are over $(100 \times 9 \times 3)$. This indicates that IDE has a great global search ability and is more stable and consistent than DE.

(3) IDE has better runtimes than DE in most test instances, and it has a greater advantage on large-sized problems. This indicates that IDE is more efficient than DE. 


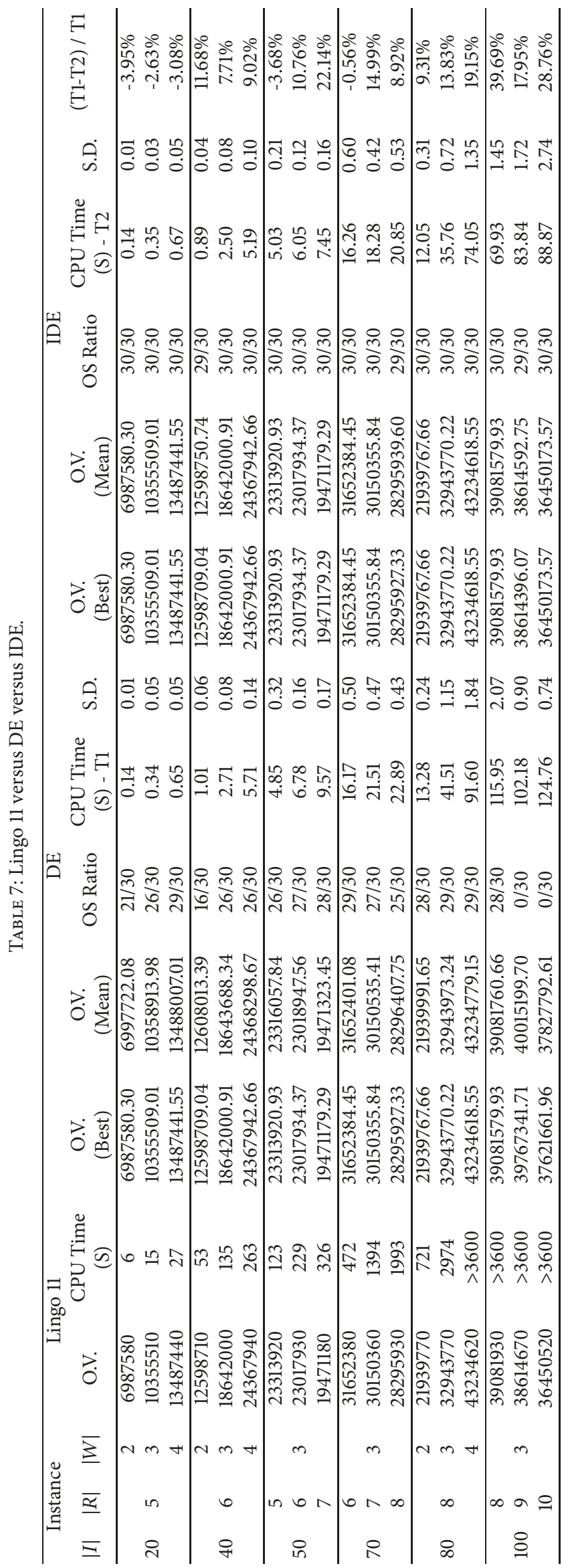


According to the comparison results, we can see that IDE is the best approach to solve MCLIPs compared with DE and Lingo 11.

\section{Conclusion and Future Research}

In this paper, we study a multicommodity location-inventory problem by considering false failure returns in a forwardreverse logistics network. To the best of our knowledge, this is the first work that considers false failure returns to optimize joint location-inventory decisions, and it contributes to the literature because of the great importance of false failure returns in practice.

This research can be extended in several directions: First, other types of returns such as defective items can be considered to make this study more practical. Second, the $(R$, $Q)$ model is adopted to replenish inventories in this work. However, there are other popular inventory control policies in practice, and it will be interesting to explore them and compare their performances in forward-reverse logistics networks. Third, it will be valuable to study location-inventoryrouting problems by considering false failure returns.

\section{Conflicts of Interest}

The authors declare that they have no conflicts of interest.

\section{Acknowledgments}

This research was supported by the National Natural Science Foundation of China under Grants nos. 71672074 and 71772075 .

\section{References}

[1] V. D. R. Guide Jr., G. C. Souza, L. N. van Wassenhove, and J. D. Blackburn, "Time value of commercial product returns," Management Science, vol. 52, no. 8, pp. 1200-1214, 2006.

[2] Z. Hua, H. Hou, and Y. Bian, "Optimal Shipping Strategy and Return Service Charge under No-Reason Return Policy in Online Retailing," IEEE Transactions on Systems, Man, and Cybernetics: Systems, vol. 47, no. 12, pp. 3189-3206, 2017.

[3] J. Stock, T. Speh, and H. Shear, "Many happy (product) returns," Harvard Business Review, vol. 80, no. 7, p. 16, 2002.

[4] M. Ferguson, V. D. R. Guide Jr., and G. C. Souza, "Supply chain coordination for false failure returns," Manufacturing and Service Operations Management, vol. 8, no. 4, pp. 376-393, 2006.

[5] T. P. Harrison, The Practice of Supply Chain Management: Where Theory And Application Converge, Springer Science \& Business Media, 2005.

[6] M. S. Pishvaee, F. Jolai, and J. Razmi, "A stochastic optimization model for integrated forward/reverse logistics network design," Journal of Manufacturing Systems, vol. 28, no. 4, pp. 107-114, 2009.

[7] M. S. Daskin, C. R. Coullard, and Z. J. M. Shen, "An inventorylocation model: formulation, solution algorithm and computational results," Annals of Operations Research, vol. 110, pp. 83$106,2002$.

[8] R. Z. Farahani, H. Rashidi Bajgan, B. Fahimnia, and M. Kaviani, "Location-inventory problem in supply chains: A modelling review," International Journal of Production Research, vol. 53, no. 12, pp. 3769-3788, 2015.

[9] Z.-J. M. Shen, C. R. Coullard, and M. S. Daskin, "A joint location-inventory model," Transportation Science, vol. 37, no. 1, pp. 40-55, 2003.

[10] L. Ozsen, M. S. Daskin, and C. R. Coullard, "Facility location modeling and inventory management with multisourcing," Transportation Science, vol. 43, no. 4, pp. 455-472, 2009.

[11] M. Amiri-Aref, W. Klibi, and M. Z. Babai, "The multi-sourcing location inventory problem with stochastic demand," European Journal of Operational Research, vol. 266, no. 1, pp. 72-87, 2018.

[12] F. Silva and L. Gao, "A Joint Replenishment Inventory-Location Model," Networks and Spatial Economics, vol. 13, no. 1, pp. 107122,2013

[13] Q. Hui, W. Lin, and L. Rui, "A contrastive study of the stochastic location-inventory problem with joint replenishment and independent replenishment," Expert Systems with Applications, vol. 42, no. 4, pp. 2061-2072, 2015.

[14] S. M. Mousavi, N. Alikar, S. T. A. Niaki, and A. Bahreininejad, "Optimizing a location allocation-inventory problem in a twoechelon supply chain network: a modified fruit fly optimization algorithm," Computers \& Industrial Engineering, vol. 87, pp. 543-560, 2015.

[15] M. a. Schuster Puga and J.-S. Tancrez, "A heuristic algorithm for solving large location-inventory problems with demand uncertainty," European Journal of Operational Research, vol. 259, no. 2, pp. 413-423, 2017.

[16] A. Diabat, E. Dehghani, and A. Jabbarzadeh, "Incorporating location and inventory decisions into a supply chain design problem with uncertain demands and lead times," Journal of Manufacturing Systems, vol. 43, pp. 139-149, 2017.

[17] J. Shu, Q. Ma, and S. Li, "Integrated location and two-echelon inventory network design under uncertainty," Annals of Operations Research, vol. 181, pp. 233-247, 2010.

[18] M. Farahani, H. Shavandi, and D. Rahmani, "A locationinventory model considering a strategy to mitigate disruption risk in supply chain by substitutable products," Computers \& Industrial Engineering, vol. 108, pp. 213-224, 2017.

[19] A. Diabat, T. Abdallah, and A. Henschel, "A closed-loop location-inventory problem with spare parts consideration," Computers \& Operations Research, vol. 54, pp. 245-256, 2015.

[20] J. Asl-Najafi, B. Zahiri, A. Bozorgi-Amiri, and A. TaheriMoghaddam, "A dynamic closed-loop location-inventory problem under disruption risk," Computers \& Industrial Engineering, vol. 90, pp. 414-428, 2015.

[21] O. Kaya and B. Urek, "A mixed integer nonlinear programming model and heuristic solutions for location, inventory and pricing decisions in a closed loop supply chain," Computers \& Operations Research, vol. 65, pp. 93-103, 2016.

[22] H. Guo, C. Li, Y. Zhang, C. Zhang, and Y. Wang, "A Nonlinear Integer Programming Model for Integrated Location, Inventory, and Routing Decisions in a Closed-Loop Supply Chain," Complexity, vol. 2018, pp. 1-17, 2018.

[23] H. Guo, C. Li, Y. Zhang, C. Zhang, and M. Lu, "A LocationInventory Problem in a Closed-Loop Supply Chain with Secondary Market Consideration," Sustainability, vol. 10, no. 6, p. 1891, 2018.

[24] H. Guo, Y. Zhang, C. Zhang, Y. Liu, and Y. Zhou, "Locationinventory decisions for closed-loop supply chain management in the presence of the secondary market," Annals of Operations Research. 
[25] M. S. Pishvaee, R. Z. Farahani, and W. Dullaert, "A memetic algorithm for bi-objective integrated forward/reverse logistics network design," Computers \& Operations Research, vol. 37, no. 6, pp. 1100-1112, 2010.

[26] S. M. Hatefi and F. Jolai, "Robust and reliable forward-reverse logistics network design under demand uncertainty and facility disruptions," Applied Mathematical Modelling, vol. 38, no. 9-10, pp. 2630-2647, 2014.

[27] S. Axsäter, "Using the deterministic EOQ formula in stochastic inventory control," Management Science, vol. 42, no. 6, pp. 830834, 1996.

[28] G. D. Eppen, "Note-Effects of Centralization on Expected Costs in a Multi-Location Newsboy Problem," Management Science, vol. 25, no. 5, pp. 498-501, 1979.

[29] Z.-J. M. Shen, "Integrated supply chain design models: a survey and future research directions," Journal of Industrial and Management Optimization, vol. 3, no. 1, pp. 1-27, 2007.

[30] R. Storn and K. Price, "Differential evolution-a simple and efficient heuristic for global optimization over continuous spaces," Journal of Global Optimization, vol. 11, no. 4, pp. 341359, 1997.

[31] B. Alatas, E. Akin, and A. Karci, "MODENAR: Multi-objective differential evolution algorithm for mining numeric association rules," Applied Soft Computing, vol. 8, no. 1, pp. 646-656, 2008.

[32] R. S. Rahnamayan, H. R. Tizhoosh, and M. M. A. Salama, "Opposition-based differential evolution," IEEE Transactions on Evolutionary Computation, vol. 12, no. 1, pp. 64-79, 2008. 


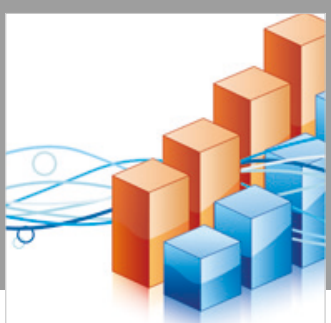

Advances in

Operations Research

\section{-n-m}
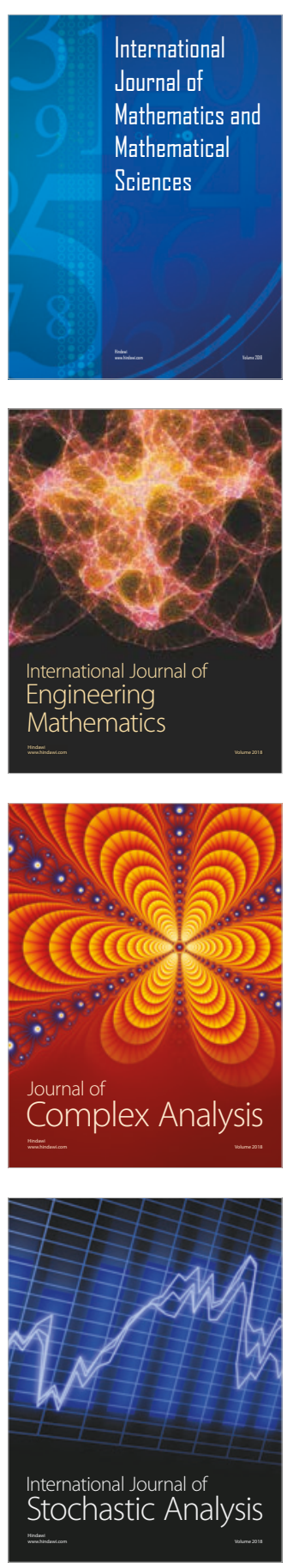
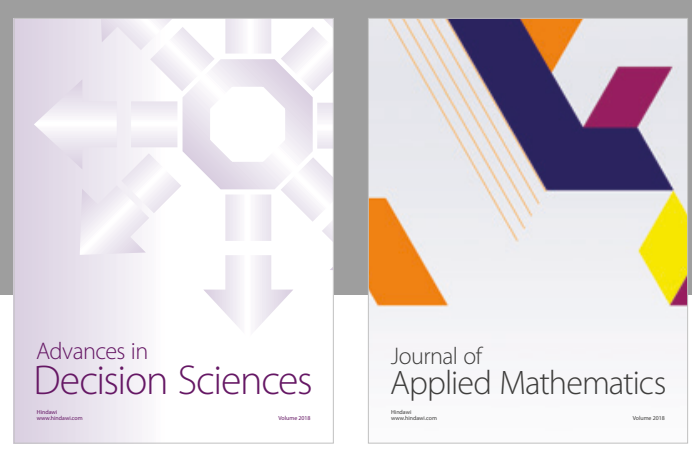

Journal of

Applied Mathematics
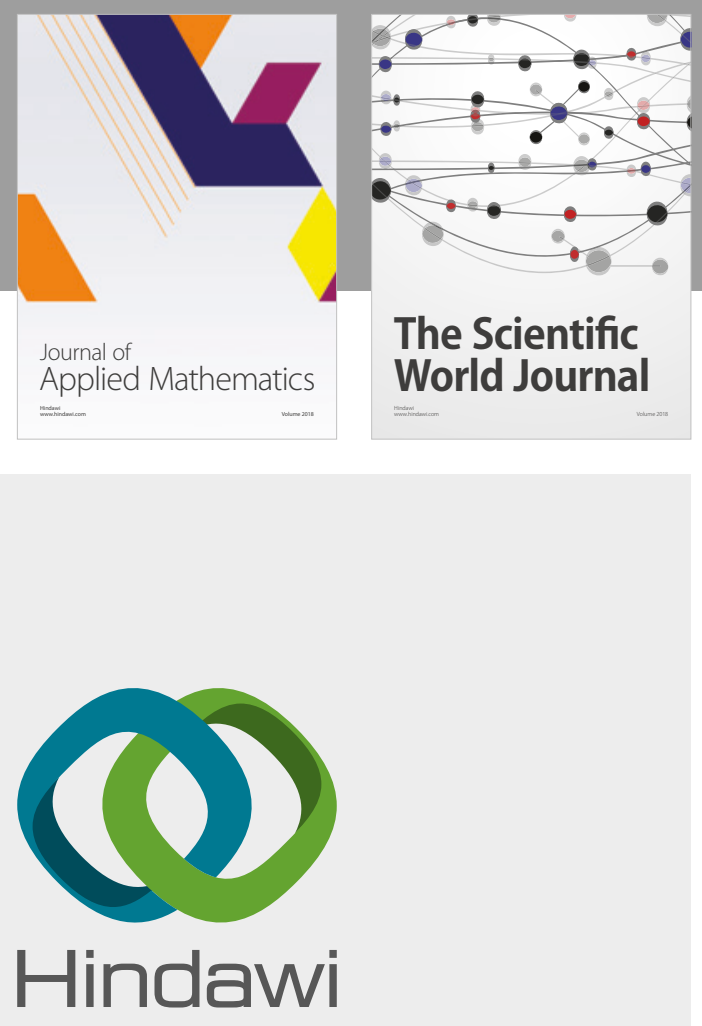

Submit your manuscripts at

www.hindawi.com

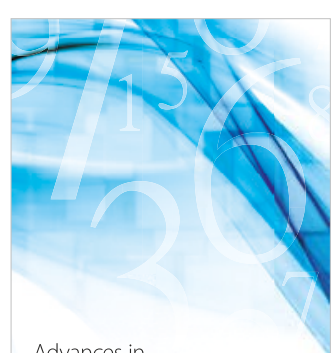

Advances in
Numerical Analysis
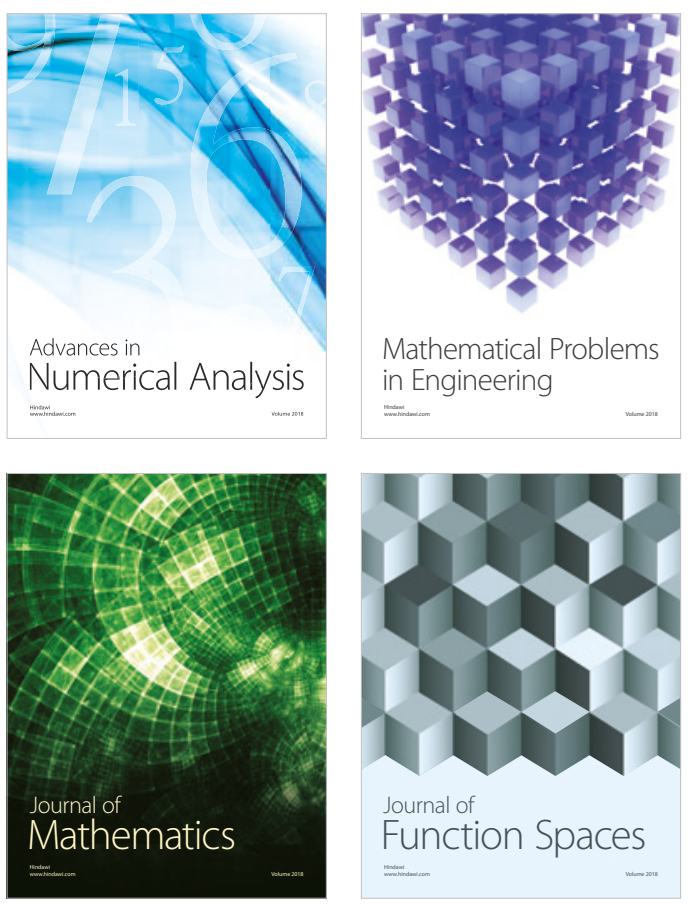

Mathematical Problems in Engineering

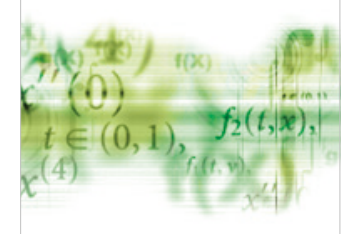

International Journal of

Differential Equations

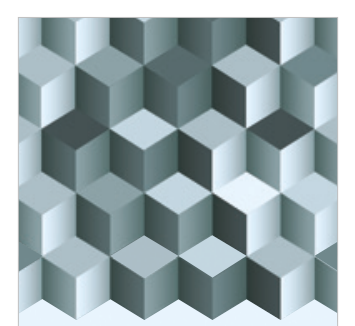

Journal of

Function Spaces
The Scientific

World Journal

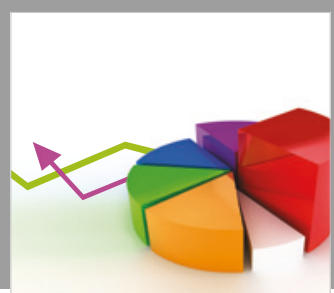

Journal of

Probability and Statistics
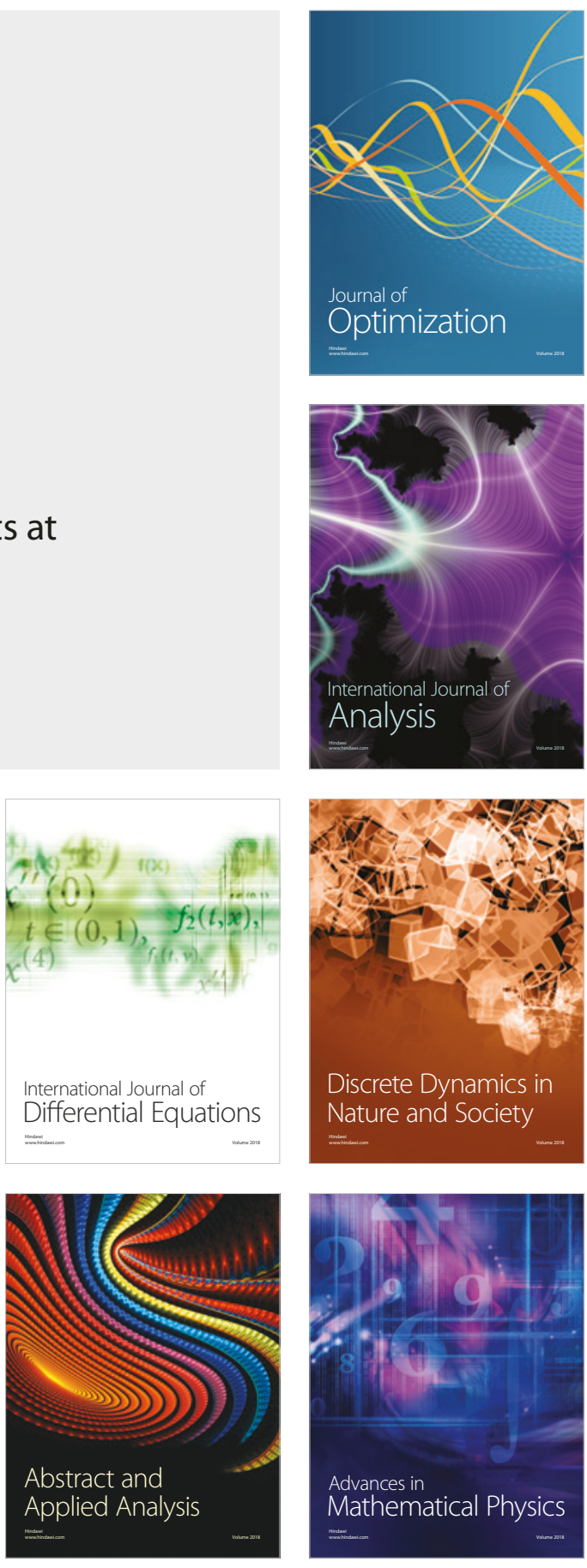